\title{
LIE ALGEBRA REPRESENTATIONS OF DIMENSION $<p^{2}$
}

\author{
HELMUT STRADE
}

\begin{abstract}
Various methods of representation theory of modular Lie algebras are improved. As an application the structure of the Lie algebras having a faithful irreducible module of dimension $<p^{2}$ is determined. Applications to the classification theory of modular simple Lie algebras are given.
\end{abstract}

\section{INTRODUCTION}

In this note we investigate finite dimensional Lie algebras over an algebraically closed field $F$ of characteristic $p>3$, which admit a faithful irreducible representation of dimension $<p^{2}$. This problem arises in a more specific form in the classification theories both of Block-Wilson [6] and Benkart-Osborn [2]. They derive the structure of these algebras under various additional assumptions, which naturally arise in the context of classification theory. Our more general approach yields some structural insight, which in turn simplifies those proofs and should also be useful for further progress in the classification theory.

In $[10,11,12]$ semisimple Lie algebras having a faithful representation of dimension less than $p$ have been fully determined. One cannot expect to get results of the same completeness in the more general situation, since the mass of examples makes it hard to describe them by means of general concepts. This reflects the fact that the strong argument on traces fails to hold, whenever the module under consideration has dimension $\geq p$.

In $\S I$ we list results and methods which have been developed during the last twenty years and now enable us to attack the problem. Some of them were improved. In $\S I I$ an appropriate (but unusual) concept of a socle is introduced. We describe the structure of the investigated Lie algebras in terms of this socle. In §III we restrict ourselves to Lie algebras of toral rank one with respect to some Cartan subalgebra (CSA) $H$ and then obtain a complete determination of all these algebras.

Received by the editors September 19, 1988.

1980 Mathematics Subject Classification (1985 Revision). Primary 17B05, 17B10, $17 \mathrm{~B} 50$.

Partially supported by NSF grant no. DMS-8702928. 
This paper was written while the author was visiting the University of Wisconsin. I would like to take the opportunity and thank Georgia M. Benkart and J. Marshall Osborn for their hospitality and support.

\section{General Results}

In this section we will for convenience state and prove some results which we will refer to in the sequel. These results describe the structure of Lie algebras and modules in the presence of some (quite natural) assumptions. We always consider Lie algebras $L$ finite dimensional over an algebraically closed ground field $F$ of characteristic $p>3$.

Theorem 1. Let $S_{1}, S_{2}$ be ideals of $L$ such that

$$
L=S_{1}+S_{2}, \quad\left[S_{1}, S_{2}\right]=0 .
$$

Assume that $\rho: L \rightarrow g l(U)$ is an irreducible representation and let $\rho_{i}: S_{i} \rightarrow$ $g l\left(U_{i}\right)$ be irreducible subrepresentations for $S_{1}, S_{2}$. Then $U \cong U_{1} \otimes U_{2}$ and $\rho \cong \rho_{1} \otimes \mathrm{id}+\mathrm{id} \otimes \rho_{2}$.

Proof. Let $A, B, C$ denote the associative subalgebras of $\operatorname{End}(U)$ generated by $\rho(L), \rho\left(S_{1}\right), \rho\left(S_{2}\right)$, respectively. Our present assumptions imply that

$$
A=\operatorname{End}(U), \quad B C=A, \quad[B, C]=0 .
$$

Let $R$ be the nilpotent radical of $B$. Then $R A=R C$ is according to $(*)$ a nilpotent two-sided ideal of $A$. As $A$ is simple this shows that $R=(0)$, proving that $B$ is semisimple. (*) also shows that the center $C(B)$ of $B$ is contained in the center of $A$, which is $F$ id. Hence $C(B)=F$ id and therefore $B$ is a central simple subalgebra of $A$ with $C(B)=C(A)$.

Put $D:=\{x \in A \mid[x, B]=0\}$. The double centralizer theorem implies that $D$ is simple and that $A \cong B \otimes_{F} D$. Since $C \subset D$ and $B C=A=B D$, a dimension argument proves $C=D$ and $A \cong B \otimes_{F} C$.

Let $B_{1}, C_{1}$ be minimal left ideals of $B$ and $C$, respectively. Then $B_{1} \otimes C_{1}$ is a minimal left ideal of $B \otimes C$. As an irreducible $B \otimes C$-module, $U$ is module-isomorphic to $B_{1} \otimes C_{1}$. With the same reasoning, any irreducible $S_{1}$ submodule of $U$ is an irreducible $B$-module isomorphic to $B_{1}$ as a $B$-module. This proves the result.

In [4] Block and Zassenhaus proved a similar result for the direct sum of Lie algebras.

Recall the Blattner-Dixmier theorem on induced representations. Although this theorem is stated in [7] only for Lie algebras over a field of characteristic 0 , Block-Wilson [6] have pointed out that it is true with only the obvious changes for the following situation:

Let $L$ be a restricted Lie algebra, $I$ an ideal of $L$ and $\rho: L \rightarrow g l(U)$ an irreducible representation. Then $\rho$ admits a character $S \in L^{*}$ such that

$$
\rho(x)^{p}-\rho\left(x^{[p]}\right)=S(x)^{p} \text { id } \forall x \in L .
$$

Let $U_{0}$ be an irreducible $I$-submodule of $U$ and put $\widetilde{U_{0}(I)}$ the sum of all $I$-submodules of $U$ isomorphic to $U_{0}$. Put

$$
G=G\left(I, U_{0}\right):=\operatorname{stab}\left(L, \widetilde{U_{0}(I)}\right)=\left\{x \in L \mid \rho(x) \widetilde{U_{0}(I)} \subset \widetilde{U_{0}(I)}\right\} .
$$


For a restricted Lie algebra $L$ and $S \in L^{*}$ we consider the ideal $J$ of $U(L)$ which is generated by the set of (central) elements $\left\{x^{p}-x^{[p]}-S(x)^{p} 1 \mid x \in L\right\} \subset$ $U(L)$. Put

$$
U(L, S):=U(L) / J .
$$

If $L$ is finite dimensional then $\operatorname{dim} U(L, S)=p^{\operatorname{dim} L} . U(L, S)$ has the following universal property: Every representation $\rho: L \rightarrow g l(U)$ which has the character $S$ (i.e. $\rho(x)^{p}-\rho\left(x^{[p]}\right)=S(x)^{p}$ id $\forall x \in L$ ) can be uniquely extended to an associative unitary representation

$$
\hat{\rho}: U(L, S) \rightarrow \operatorname{End}(U) .
$$

Let $G$ be a restricted subalgebra of $L$ of finite codimension. Then $U(L, S)$ is a free right $U(G, S \mid G)$-module of rank $p^{\operatorname{dim} L / G}$. (For the general notation we always refer to [15].)

Theorem 2 (Blattner, Dixmier). Let $L$ be a restricted Lie algebra, $U$ an irreducible L-module of character $S, I$ an ideal of $L, U_{0}$ an irreducible $I$ submodule and $G=G\left(I, U_{0}\right)$. Then

(1) $G$ is a restricted subalgebra of $L$.

(2) $U \cong U(L, S) \otimes_{U(G, S \mid G)} \widetilde{U_{0}(I)}$.

(3) $\widetilde{U_{0}(I)}$ is an irreducible G-module.

For further references we state the following corollary.

Theorem 3. Let $L$ be a (nonrestricted) Lie algebra and I an ideal of $L$. Suppose $L$ has a faithful irreducible representation $\rho: L \rightarrow g l(U)$ with $\operatorname{dim} U<p^{2}$. Let $U_{0}$ be an irreducible I-submodule of $U$. Then $\operatorname{dim} \widetilde{U_{0}(I)}<p$ or $U_{0}$ is a faithful I-module.

Proof. Let $L_{p}$ denote the $p$-envelope of $\rho(L)$ in $g l(U)$. Note that $\rho(I)$ is an ideal of $L_{p}$. If $\operatorname{dim} \widetilde{U_{0}(I)} \geq p$. then since $\operatorname{dim} U<p^{2}$ Theorem 2 yields $U=$ $U_{0}(I)$. Therefore $U=\sum W_{i}$ is the sum of irreducible $I$-modules isomorphic to $U_{0}$. If $x \in I$ annihilates $U_{0}$, then

$$
\rho(x)(U)=\sum \rho(x)\left(W_{i}\right)=0 .
$$

The faithfulness of $\rho$ gives the result.

If in Theorem 2 the ideal $I$ is assumed to be abelian we have the following more detailed description. Let $L_{p}$ denote the $p$-envelope of $\rho(L)$ in $g l(U)$.

Lemma 4. Let $I$ be an ideal of a (nonrestricted) Lie algebra $L$ and assume that $\rho: L \rightarrow g l(U)$ is an irreducible L-module.

(1) If I acts nilpotently on some nonzero I-submodule then $I \subset \operatorname{ker}(\rho)$.

(2) If $I^{(1)} \subset \operatorname{ker}(\rho)$ then there exists $E \in I^{*}$ such that for all $x \in I, \rho(x)-$ $E(x)$ id is nilpotent.

(3) Assume that $I^{(1)} \subset \operatorname{ker}(\rho)$. Then any irreducible $I$-submodule $U_{0}$ is onedimensional and $G\left(I, U_{0}\right)=\left\{g \in L_{p} \mid E([\rho(x), g])=0 \forall x \in I\right\}$. Moreover, $L_{p}=G\left(I, U_{0}\right)$ if and only if $[L, I] \subset \operatorname{ker}(\rho)$. 
(4) If $I$ is abelian and $[L, I] \not \subset \operatorname{ker}(\rho)$ then $\operatorname{dim} U \geq p \operatorname{dim} \widetilde{U_{0}(I)}$.

Proof. (1) Since $I$ acts nilpotently on some $I$-submodule $U^{\prime} \neq(0)$, there is a nonzero vector annihilated by $\rho(I)$. Put $U_{0}=\{u \in U \mid \rho(x) u=0 \forall x \in I\}$. This is an $L$-submodule and nonzero. Hence $U_{0}=U$, proving $I \subset \operatorname{ker}(\rho)$.

(2) Decompose $U$ into weight spaces with respect to $\rho(I)$. Since $I$ is an ideal and hence acts nilpotently on $\rho(L)$ these weight spaces are $L$-submodules. The irreducibility of $U$ entails the existence of some mapping $E: I \rightarrow F$ such that $\rho(x)-E(x)$ id is nilpotent for all $x \in I$. Since $\rho(I)$ is abelian there is a common eigenvector $u \neq 0$. Then

$$
E(x+\alpha y) u=\rho(x+\alpha y) u=\rho(x) u+\alpha \rho(y) u=E(x) u+\alpha E(y) u
$$

for all $x, y \in I, \alpha \in F$. Therefore $E$ is linear.

(3) Clearly $U_{0}$ is one-dimensional and

$$
\widetilde{U_{0}(I)}=\{u \in U \mid \rho(x) u=E(x) u \forall x \in I\} .
$$

Take any $g \in G\left(I, U_{0}\right)=\left\{h \in L_{p} \mid h \widetilde{U_{0}(I)} \subset \widetilde{U_{0}(I)}\right\}$. Then we have for $u \in$ $\widetilde{U_{0}(I)}$ and $x \in I$

$$
\rho(x)(g \cdot u)=E(x) g \cdot u=g \cdot E(x) u=g \cdot \rho(x) u
$$

proving $[\rho(x), g] u=0$ and $E([\rho(x), g])=0$. Next assume $E([\rho(x), g])=$ 0 for some $g \in L_{p}$. Then $[\rho(x), g] u=0 \forall x \in I, u \in U_{0}(I)$ and hence $\rho(x)(g \cdot u)=E(x) g \cdot u$. Therefore $g \cdot u \in \widetilde{U_{0}(I)}$ and $g \in G\left(I, U_{0}\right)$.

If $L_{p}=G\left(I, U_{0}\right)$ then $[L, I] \subset \operatorname{ker}(E) .[L, I]$ is an ideal of $L$ contained in $\operatorname{ker}(E)$. Hence it acts nilpotently on $U$. According to (1) we obtain the result.

(4) Apply Theorem 2 and (3).

As a result of Lemma 4 we obtain that whenever $L$ has a noncentral abelian ideal, only the first case of Theorem 3 can occur.

Actually, we want to derive some general information about both cases. To do this we recall the following notation.

Any linear mapping $\varphi: L \rightarrow \operatorname{End}(U)$ is said to be a projective representation, if $\varphi([x, y])-[\varphi(x), \varphi(y)] \in F$ id for all $x, y \in L$.

Let $A(n ; \mathbf{1})$ denote the truncated polynomial ring

$$
F\left[X_{1}, \ldots, X_{n}\right] /\left(X_{1}^{p}, \ldots, X_{n}^{p}\right)
$$

and $W(n ; \mathbf{1})=\operatorname{Der}(A(n ; \mathbf{1}))$ the restricted Jacobson-Witt algebra.

For any finite dimensional associative algebra $A, \operatorname{Jac}(A)$ denotes the maximal nilpotent ideal of $A$.

The following improves a result of [13].

Theorem 5. Let $I$ be an ideal of the restricted Lie algebra L. Assume that $\rho: L \rightarrow g l(U)$ is an irreducible representation. Let $U_{0}$ be an irreducible Isubmodule and put $B$ equal to the subalgebra of $\operatorname{End}(U)$ generated by $\rho(I)$. The following cases can occur: 
(1) $B$ is simple. Then $U \cong U_{0} \otimes_{F} U_{1}$, where $U_{1}$ is an irreducible projective module of $L / I$, and $L=G\left(I, U_{0}\right)$. B acts on $U$ via

$$
b \cdot\left(u_{0} \otimes u_{1}\right)=\left(b \cdot u_{0}\right) \otimes u_{1} \quad \forall b \in B, u_{0} \in U_{0}, u_{1} \in U_{1} .
$$

(2) $B$ is not simple. Then $B / \operatorname{Jac}(B)$ is simple. In addition, $L \neq G\left(I, U_{0}\right)$. Proof. Let $A=\operatorname{End}(U)$ be the algebra generated by $\rho(L)$. Since $I$ is an ideal of $L$, the multiplication in $L$ extends to a mapping $\mu: L \rightarrow \operatorname{Der}(B)$ determined by $\mu(x)(y)=[\rho(x), y] \quad \forall x \in L, y \in B$.

(1) Assume that $B$ is simple and $C(B)=C(A)$. Then the double centralizer theorem proves that $C:=\{a \in A \mid[a, B]=0\}$ is a simple algebra and $A \cong$ $B \otimes C$, i.e. $B C=A, B \cap C=F$ id. Since any derivation of $B$ is inner, there is a linear mapping

$$
f: L \rightarrow B
$$

such that $[\rho(x), b]=[f(x), b] \quad \forall x \in L, \forall b \in B$. Then $\rho(x)-f(x) \in C$ proving $\rho(L) \subset B+C$. As $\rho(I) \subset B$ there is a Lie algebra homomorphism

$$
\varphi: L / I \rightarrow(B+C) / B \cong C / F \text { id } .
$$

Let $L^{\prime}$ be the preimage of $\varphi(L / I)$ in $C . L^{\prime}$ is an extension of $\varphi(L / I)$ by the one-dimensional center $F$ id. $C$ is generated by $L^{\prime}$ as $L$ generates $A$.

Let $B_{1}, C_{1}$ be minimal left ideals of $B$ and $C$, respectively. Then $U \cong$ $B_{1} \otimes C_{1}$ and $U_{0} \cong B_{1}$. The above also shows that $C_{1}$ is an irreducible projective $L / I$-module. Since $B$ is simple, $U$ decomposes into a direct sum of irreducible modules isomorphic to $U_{0}$. Theorem 2 yields $L=G\left(I, U_{0}\right)$.

(2) We now consider the general case. Let $R$ denote the maximal nilpotent $\mu(L)$-invariant ideal of $B$. Then $R A$ is a two-sided nilpotent ideal of $A$, whence $R=0$. Therefore $B$ is $\mu(L)$-semisimple. From [5] we conclude that there exist simple algebras $B_{1}, \ldots, B_{k}$ and $n_{1}, \ldots, n_{k}$ such that

$$
B \cong \bigoplus_{i=1}^{k} B_{i} \otimes A\left(n_{i} ; \mathbf{1}\right)
$$

Moreover, any $B_{i} \otimes A\left(n_{i} ; \mathbf{1}\right)$ is $\mu(L)$-invariant and

$$
\operatorname{Der}\left(B_{i} \otimes A\left(n_{i} ; \mathbf{1}\right)\right)=\operatorname{Der}\left(B_{i}\right) \otimes A\left(n_{i} ; \mathbf{1}\right)+F \otimes W\left(n_{i} ; \mathbf{1}\right) .
$$

Since $B_{i}$ is simple, we obtain $\mu(L) \subset \operatorname{Der}\left(B_{i} \otimes A\left(n_{i} ; \mathbf{1}\right)\right) \cong \operatorname{ad} B_{i} \otimes A\left(n_{i} ; \mathbf{1}\right)+$ $F \otimes W\left(n_{i} ; \mathbf{1}\right)$. This proves that $\mu(L)$ annihilates $\bigoplus_{i=1}^{k} C\left(B_{i}\right) \otimes F$. Since $\rho(L)$ generates $A$, this means $\bigoplus_{i=1}^{k} C\left(B_{i}\right) \otimes F \subset C(A)=F$ id. As a result, $k=1$ and $B=B_{1} \otimes A(n ; \mathbf{1})$ (for $n=n_{1}$ ) as well as $C\left(B_{1}\right)=C(A)$.

Put $D:=\left\{x \in A \mid\left[x, B_{1}\right]=0\right\}$. As above $A \cong B_{1} \otimes D$. Note that

$$
\sum B_{1} \otimes F \prod x_{i}^{s_{i}}\left(s_{1}+\cdots+s_{n} \geq 1\right)
$$

is a nilpotent ideal of $B_{1} \otimes A(n ; 1)$ proving that $B_{1} \cong B / \mathrm{Jac}(B)$ is simple. Now assume that (1) is not true. Then $n \neq 0$ and $\operatorname{Jac}(B) \neq 0$. Since $U$ is a 
faithful $B$-module, it is not the direct sum of irreducible $B$-modules. Therefore $\widetilde{U_{0}(I)} \neq U$. According to Theorem 2 this yields $L \neq G\left(I, U_{0}\right)$.

This theorem reduces the representation theory of Lie algebras with a noncentral ideal to representations of smaller algebras.

We also may apply the first part of the theorem to the second: Let $\rho: L \rightarrow$ $g l(U)$ be an irreducible representation of the restricted algebra $L$ and assume that $I$ is an ideal of $L$ such that $L \neq G\left(I, U_{0}\right):=G$ for some $I$-submodule $U_{0}$. Then Theorem 2 proves that $U$ is induced from the irreducible $G$-module $\widetilde{U_{0}(I)}$. Applying Theorem 5 to $\rho: G \rightarrow g l\left(\widetilde{U_{0}(I)}\right)$, we see that the first case occurs. Hence $\tilde{U}_{0}(I)$ is the tensor product of $U_{0}$ with some irreducible projective $G / I$-module.

The problem, how to describe restricted Lie algebras having a suitable large subalgebra (which occurs if $L \neq G\left(I, U_{0}\right)$ ), was dealt with in [9]. Recall that

$$
W(n ; \mathbf{1})_{(k)}=\sum_{i=1}^{n} \sum_{|a| \geq k+1} F x^{a} \partial_{i}
$$

denotes the canonical filtration of $W(n ; \mathbf{1})$.

Definition [9]. A subalgebra $S$ of $W(n ; \mathbf{1})$ is called essential, if $S+W(n ; \mathbf{1})_{(0)}$ $=W(n ; \mathbf{1})$.

Theorem 6 [9]. Let $L$ be a restricted Lie algebra and $G$ a restricted subalgebra of codimension $n$. Then there exists a restricted homomorphism

$$
\varphi: L \rightarrow W(n ; \mathbf{1})
$$

such that

(1) $\varphi(L)$ is an essential subalgebra of $W(n ; \mathbf{1})$.

(2) $\operatorname{ker}(\varphi)$ is the unique maximal ideal of $L$ which is contained in $G$.

(3) $\varphi(L) \cap W(n ; \mathbf{1})_{(0)}=\varphi(G)$.

It will come out that in this note the essential subalgebras of $W(1 ; 1)$ are of major importance. We improve the results on these algebras given in [9].

Proposition 7. Suppose that $L$ is an essential subalgebra of $W(1 ; \mathbf{1})$. Then either $L$ coincides with $W(1 ; \mathbf{1})$ or there exists an automorphism $\varphi$ of $W(1 ; \mathbf{1})$ such that $\varphi(L) \subset F \partial+F x \partial+F x^{2} \partial$. In either case $L$ is a restricted subalgebra of $W(1 ; \mathbf{1})$.

Proof. We consider the filtration of $L$ inherited by the filtration of $W(1 ; \mathbf{1})$. Let $i:=\max \left\{j \mid L \cap W(1 ; \mathbf{1})_{(j)} \neq 0\right\}$. If $i \geq 2$ then, as $L$ is essential and therefore contains an element $\partial+f, f \in W(1 ; \mathbf{1})_{(0)}$, and $L+W(1 ; \mathbf{1})_{(3)}=$ $W(1 ; \mathbf{1})$. It is easily seen that $L=W(1 ; \mathbf{1})$ in this case.

If $i=-1$, then $L=F e$ with $e=\partial+\sum_{i=1}^{p-1} \alpha_{i} x^{i} \partial$. Applying an automorphism $\exp (a d y)$ for some $y \in W(1 ; 1)_{(2)}$, we may assume $e=\partial+\alpha x \partial$. If $i=0,1$ there exists an element $h \in L \cap W(1 ; \mathbf{1})_{(0)}$, which does not act 
nilpotently on $W(1 ; \mathbf{1}) . F h$ is then a CSA of $W(1 ; \mathbf{1})$. Adjusting $h$ by some nonzero scalar we can construct a proper basis $f_{-1}, \ldots, f_{p-2}$ of $W(1 ; \mathbf{1})$ such that $f_{0}=h$ and $f_{-1} \in L$. The mapping $\varphi: f_{i} \mapsto x^{i+1} \partial$ defines an automorphism of $W(1 ; \mathbf{1})$.

This proves the first assertion. $F \partial+F x \partial+F x^{2} \partial$ is a restricted subalgebra of $W(1 ; \mathbf{1})$ and isomorphic to $\operatorname{sl}(2)$. The subalgebras of $\operatorname{sl}(2)$ are: onedimensional abelian, two-dimensional solvable nonabelian, three-dimensional simple. Hence the $p$-closure of $\varphi(L)$ has the same dimension as $\varphi(L)$. This shows that $\varphi(L)$ is a restricted subalgebra of $W(1 ; \mathbf{1})$. Since $W(1 ; \mathbf{1})$ is centerless, every automorphism is a restricted mapping.

\section{STRUCTURE THEORY OF LIE ALGEBRAS WITH FAITHFUL IRREDUCIBLE MODULE OF DIMENSION $<p^{2}$}

Semisimple Lie algebras having a faithful representation of dimension $<p$ have been determined in $[10,11,12]$. We improve on the results of those papers. (We will refer to a previous chapter as usual: (I.4) means Lemma 4 in $\S I$.)

Theorem 1. Let $L$ be any Lie algebra and $\rho: L \rightarrow g l(U)$ a faithful irreducible representation of dimension $<p$. Then

(1) $L=\bigoplus_{i=1}^{k} A_{i} \oplus C(L)$ where either all $A_{i}$ are classical simple algebras or $k=1$ and $A_{1}=W(1 ; \mathbf{1})$.

(2) $\operatorname{Der}\left(A_{i}\right)=\operatorname{ad} A_{i}$ in all cases.

(3) Let $U_{i}$ be irreducible $A_{i}$-submodules $(i=1, \ldots, k)$. Then $U \cong U_{1} \otimes$ $\cdots \otimes U_{k}$.

Proof. (1) Let $x \in L^{(1)} \cap C(L)$. As $U$ is irreducible, $x$ acts as a scalar, $\rho(x)=\alpha$ id. Since $x \in L^{(1)}$ we have

$$
0=\operatorname{trace}(\rho(x))=\alpha \operatorname{dim} U,
$$

i.e. $\alpha=0$. This shows $L^{(1)} \cap C(L)=(0)$. There exists an ideal $S$ of $L$ containing $L^{(1)}$ such that $L=S \oplus C(L), C(S)=0$. Assume that $S$ has an abelian ideal $I \neq 0$. According to (I.4) this implies $\operatorname{dim} U \geq p$, a contradiction. This proves $\operatorname{rad}(S)=0$. We can now apply the results of $[10,11,12]$ and obtain (1).

(2) If $A_{1}=W(1 ; \mathbf{1})$ the result is known. If $A_{i}$ is classical and by assumption has a faithful irreducible module of dimension $<p$ it has a nondegenerate trace form. Then all derivations are inner [3]. (3) results from (I.(1)).

Recall that a Lie algebra $L$ is said to be indecomposable, if it is not a direct sum of ideals:

$$
L=S_{1} \oplus S_{2} \Rightarrow S_{1}=0 \text { or } S_{2}=0 .
$$

Corollary. Suppose $\rho: L \rightarrow g l(U)$ is a faithful irreducible representation. Let $S$ be an ideal of $L$ having a submodule $U_{0}$ of dimension $<p$. Then $S^{(1)} \cap C(L)=$ 
0 . If moreover $L$ is indecomposable and $U_{0}$ irreducible faithful, then either $S=L$ and $C(L)=0$ or $\operatorname{dim} S=1$.

Proof. Let $V \subset U_{0}$ be an irreducible $S$-submodule with representation $\mu: S \rightarrow$ $g l(V)$. Theorem (II.1) applies to $S / \operatorname{ker}(\mu)$, proving

$$
C\left((S / \operatorname{ker}(\mu))^{(1)}\right)=0 .
$$

This implies $C(L) \cap S^{(1)} \subset \operatorname{ker}(\mu)$. Put in (I.4) $I=C(L) \cap S^{(1)}$. Then (I.4(1)) yields $C(L) \cap S^{(1)}=0$. Next assume that $U_{0}$ is faithful. According to Theorem (II.1) we have $S^{(1)}=\bigoplus_{i=1}^{k} A_{i}, A_{i}$ simple and $S=S^{(1)} \oplus C(S)$. Let $D$ be any derivation of $S$. Then $D\left(A_{i}\right)=D\left(\left(A_{i}\right)^{(1)}\right) \subset\left[D\left(A_{i}\right), A_{i}\right] \subset A_{i}$. Therefore $D \mid A_{i}$ is a derivation, hence inner.

As a result, for any $x \in L$ there is an element $y \in S^{(1)}$ such that

$$
\left[x-y, S^{(1)}\right]=0 \text {. }
$$

Put

$$
R:=\left\{x \in L \mid\left[x, S^{(1)}\right]=0\right\} .
$$

Then $L=S^{(1)} \oplus R$. Since $L$ is indecomposable, this implies $S^{(1)}=0$ or $R=0$. In the latter case $L=S$ and $C(L)=0$. In the former case $S$ is abelian, and as it has a faithful irreducible representation this yields $\operatorname{dim} S=1$.

Throughout this section we assume from now on

(A) $L$ is an indecomposable Lie algebra having a faithful irreducible representation $\rho: L \rightarrow g l(U)$ of dimension $<p^{2}$.

We are going to construct some kind of socle $S(L)$ for $L$.

Lemma 2. Assume that $C(L) \neq 0$. Suppose that $L$ has an ideal $K_{1}$ with the properties

(a) $K_{1}^{(1)}=K_{1}$.

(b) $C(L) \subset K_{1}$.

(c) $K_{1} / C(L)$ is a minimal ideal of $L / C(L)$.

Then

(1) $C(L) \cap K_{0}^{(1)}=0$ holds for any ideal $K_{0}$ with $K_{1} \not \subset K_{0}$.

(2) $\operatorname{rad}(L)=C(L)$.

Proof. We prove both assertions simultaneously, by putting $K_{0}=\operatorname{rad}(L)$ in case of (2). Since $K_{1}$ is not solvable, we also have $K_{1} \not \subset K_{0}$ in this case.

Suppose $K_{0}$ is an ideal with $K_{1} \not \subset K_{0}$. The minimality of $K_{1} / C(L)$ implies that $K_{1} \cap K_{0} \subset C(L)$. Then

$$
\left[K_{0}, K_{1}\right]=\left[K_{0}, K_{1}^{(1)}\right] \subset\left[\left[K_{0}, K_{1}\right], K_{1}\right] \subset\left[C(L), K_{1}\right]=0 .
$$

Let $V$ be an irreducible $\left(K_{0}+K_{1}\right)$-submodule of $U$ and $U_{i}$ irreducible $K_{i}$ submodules of $V(i=0,1)$. According to (I.1) and our general assumption this yields

$$
p^{2}>\operatorname{dim} U \geq \operatorname{dim} V=\left(\operatorname{dim} U_{0}\right)\left(\operatorname{dim} U_{1}\right) .
$$


As $0 \neq C(L) \subset K_{1}^{(1)}$ the corollary to II.(1) proves $\operatorname{dim} U_{1} \geq p$ and therefore $\operatorname{dim} U_{0}<p$. Next consider the ideal $I:=K_{0}+K_{1}$, and apply (I.3). Since $\operatorname{dim} V \geq p$ this implies that $V$ is a faithful $\left(K_{0}+K_{1}\right)$-module. According to (I.1) $U_{0}$ is a faithful $K_{0}$-module. (II.1, corollary) yields $K_{0}^{(1)} \cap C(L)=0$ and we are done in case (1).

Next assume $K_{0}=\operatorname{rad}(L)$. Then $\operatorname{dim} U_{0}$ is a p-power; hence $\operatorname{dim} U_{0}=1$. Since $U_{0}$ is faithful this implies $\operatorname{dim} K_{0}=1$. Moreover, since $V$ is a faithful irreducible $\left(K_{0}+K_{1}\right)$-module, we have $\operatorname{dim} C\left(K_{0}+K_{1}\right) \leq 1$. As $C\left(K_{0}+K_{1}\right) \supset$ $C(L)+K_{0}$, this proves $K_{0} \subset C(L)$.

Let $\bar{K}_{1} \oplus \cdots \oplus \bar{K}_{t}$ be the sum of all minimal, nonabelian ideals of $L / C(L)$ and $K_{i}$ the preimage of $\bar{K}_{i}$ in $L$. Note that $\operatorname{dim} C(L) \leq 1$. If there is some $j$, say $j=1$, such that $K_{j}^{(1)} \cap C(L) \neq 0$ then (II.2) and the minimality of all $K_{i}$ prove that $C(L) \not \subset K_{i}^{(1)}$ for all $i \neq 1$. In addition, $\operatorname{rad}(L)=C(L) \subset K_{1}$ in this case. We then put $S_{1}:=K_{1}, S_{i}:=K_{i}^{(1)} i \neq 1$. If $K_{i}^{(1)} \cap C(L)=0$ for all $i$, we put $S_{1}=\operatorname{rad}(L)$ and $S_{i+1}=K_{i}^{(1)} i \geq 1$.

Finally define

$$
S(L):=\sum S_{i} .
$$

By construction $S(L)$ has the following properties.

\section{Proposition 3.}

(1) $S_{i}^{(1)}=S_{i}, C(L) \cap S_{i}=0, S_{i}$ is a minimal ideal of $L \quad(i>1)$.

(2) Either $S_{1}=\operatorname{rad}(L)$ or $C(L) \subset S_{1}^{(1)}, S_{1}^{(1)}=S_{1}, S_{1} / C(L)$ is L-irreducible.

(3) $S(L)=\bigoplus S_{i}$.

Proof. (1) For $i>1$ we have $K_{i}^{(1)} \cap C(L)=0 . K_{i} / C(L)$ is a nonabelian and minimal ideal of $L / C(L)$. Therefore $K_{i}=K_{i}^{(1)} \oplus C(L)=S_{i} \oplus C(L)$. This proves (1).

(2) Is true by definition.

(3) Let $\pi: L \rightarrow L / C(L)$ be the canonical homomorphism. If $S_{1} \neq \operatorname{rad}(L)$ then $\pi(S(L))=\bigoplus \pi\left(S_{i}\right)$ proving that

$$
S_{i} \cap \sum_{j \neq i} S_{j}=(0) \quad \forall i .
$$

If $S_{1}=\operatorname{rad}(L)$ is solvable, then $\pi\left(S_{1} \cap \sum_{j>1} S_{j}\right)$ is a solvable ideal contained in $\bigoplus_{j>1} \pi\left(S_{j}\right)=\bar{K}_{1} \oplus \cdots \oplus \bar{K}_{t}$ and therefore has to be zero. Then

$$
S_{1} \cap \sum_{j>1} S_{j} \subset C(L) \cap\left(\sum_{j>1} S_{j}\right)=0 .
$$

Since $\pi: \sum_{j>1} S_{j} \rightarrow \sum_{j>1} \pi\left(S_{j}\right)$ is an isomorphism we are done.

In order to derive the structure in more detail, we first settle two particular cases. 
Lemma 4. Let $R^{\prime}$ be a simple Lie algebra and $R:=R^{\prime} \otimes A(r ; 1)$. Put $Q_{r}=$ $\sum R^{\prime} \otimes F \prod x_{i}^{s_{t}}\left(s_{1}+\cdots+s_{n} \geq r\right)$. Then

(1) $Q_{1}$ is the unique maximal ideal of $R$.

(2) $Q_{1}$ is nilpotent.

(3) $Q_{1}^{(r)} \neq 0$ if $2^{r} \leq n(p-1)$.

(4) $Q_{r}=\left(\operatorname{ad} Q_{1}\right)^{r}(R)$.

(5) $Q_{r}$ is an ideal of $R$.

(6) $R=R^{\prime} \otimes F \oplus Q_{1}$.

Proof. $Q_{1}$ is an ideal of $R$ satisfying $R=R^{\prime} \otimes F \oplus Q_{1}$. Since $R^{\prime} \cong R / Q_{1}$ is simple, it is maximal. Note that

$$
\left[R^{\prime} \otimes F \prod x_{i}^{s_{i}}, R^{\prime} \otimes F \prod x_{i}^{t_{i}}\right]=R^{(1)} \otimes F \prod x_{i}^{s_{i}+t_{i}} .
$$

This proves (4) and hence (5) and (2). Moreover, it also implies that $Q_{1}^{(r)}=Q_{2^{r}}$ which yields (3). In order to prove (1) we consider any ideal $J$. If $J \not \subset Q_{1}$ then $R=Q_{1}+J$ and hence $R / J$ is nilpotent. Since $R^{(1)}=R$ this is possible only if $R / J=0$. Therefore $Q_{1}$ is the unique maximal ideal of $R$.

Lemma 5. (1) Assume that a Lie algebra $R$ has a faithful irreducible module of dimension $<p^{2}$. Suppose that $I$ is a nilpotent ideal. Then $I^{(2)}=0$.

(2) Let $R$ be one of the direct summands in $S(L)$. If $R$ has a faithful irreducible module of dimension $<p^{2}$ then $R / C(L)$ is simple as an algebra or $R$ is solvable.

Proof. (1) Let $\rho: R \rightarrow g l(V)$ denote the representation. Apply (I.3) and take an irreducible $I$-submodule $V_{0}$ of $V$. If $\operatorname{dim} V_{0} \geq p$ then $V_{0}$ is a faithful irreducible $I$-submodule of dimension $<p^{2}$. From [9, Corollary 3.3] we conclude $I^{(2)}=0$. If $\operatorname{dim} V_{0}<p$ then, as it is a $p$-power, $\operatorname{dim} V_{0}=1 . I^{(1)}$ annihilates $V_{0}$. (I.4) then yields $I^{(1)}=0$.

(2) Assume that $R / C(L)$ is neither simple nor solvable. Then by definition $R / C(L)$ is $L$-irreducible. Hence [5] yields the existence of $n \geq 1$ such that $R / C(L) \cong R^{\prime} \otimes A(n ; \mathbf{1})$ for some simple algebra $R^{\prime}$.

According to (II.4) $Q_{1}$ is a nilpotent ideal and $Q_{1}^{(2)} \neq 0$. Let $I$ be the preimage of $Q_{1}$ in $R$. Then $I$ is nilpotent and $I^{(2)} \neq 0$, contradicting (1).

Lemma 6. Let $R$ be one of the direct summands of $S(L)$ but $R \neq L$ and $R$ not solvable. If $U$ has an irreducible $R$-submodule $U_{0}$ of dimension $<p$, then

$$
R=R^{\prime} \otimes A(1 ; \mathbf{1})
$$

where $R^{\prime}$ is simple and has a faithful irreducible module of dimension $<p$.

Proof. (a) According to (II.1, Corollary) we may assume that $U_{0}$ is not a faithful module and that $R^{(1)} \cap C(L)=0$. Therefore $R=R^{(1)}$ is $L$-irreducible. [5] yields the existence of $n \geq 1$ such that $R=R^{\prime} \otimes A(n ; 1)$ for some simple algebra $R^{\prime}$. 
The imbedding $R^{\prime} \cong R^{\prime} \otimes F$ gives rise to a faithful representation of $R^{\prime}$ in $U_{0}$ of dimension $<p$.

(b) We may consider $L$ as contained in $g l(U)$ and put $L_{p}$ the $p$-envelope of $L$ in $g l(U) . L_{p}$ acts on $R$ as an algebra of derivations. Since according to (a) (II.1) is applicable we obtain $\operatorname{Der}\left(R^{\prime}\right) \cong$ ad $R^{\prime}$. As $\operatorname{Der}\left(R^{\prime}\right) \cong R^{\prime}$ (II.1) and $\operatorname{Der}(R)=\operatorname{Der}\left(R^{\prime}\right) \otimes A(n ; \mathbf{1})+F$ id $\otimes W(n ; \mathbf{1})$ [5], there exists a mapping $f: L_{p} \rightarrow R$ such that

$$
\left.\operatorname{ad}(b-f(b))\right|_{R} \in F \text { id } \otimes W(n ; \mathbf{1}) \quad \forall b \in L_{p} .
$$

Using (II.4(5)) this yields inductively

$$
\left(\operatorname{ad} b_{1}\right) \circ \cdots \circ\left(\operatorname{ad} b_{t}\right)\left(R^{\prime} \otimes x_{1}^{p-1} \cdots x_{n}^{p-1}\right) \subset \sum R^{\prime} \otimes R x_{1}^{r_{1}} \cdots x_{n}^{r_{n}}
$$

where $r_{1}+\cdots+r_{n} \geq n(p-1)-t, \forall b_{i} \in L_{p}$.

(c) Next we apply (I.2) with $G=G\left(R, U_{0}\right)$,

$$
U=U\left(L_{p}, S\right) \otimes_{U(G, S \mid G)} \widetilde{U_{0}(R)} .
$$

Since $U_{0}$ is assumed not to be faithful, we obtain $\operatorname{dim} \widetilde{U_{0}(R)}<p$ and $\operatorname{dim} L_{p} / G$ $=1$.

Note that in accordance with (II.1) and $R^{(1)}=R$, the solvable ideal $Q_{1}$ annihilates $U_{0}$ and therefore $\widetilde{U_{0}(R)}$. Since $Q_{1}$ is a maximal ideal of $R$ it is the exact annihilator. $\widetilde{U_{0}(R)}$ is a $G$-module. We obtain $\left[G, Q_{1}\right] \subset Q_{1}$ and therefore $\left[G, Q_{r}\right] \subset Q_{r} \forall r$. Moreover, $R^{\prime} \otimes F x_{1}^{p-1} \cdots x_{n}^{p-1}=\left(\operatorname{ad} Q_{1}\right)^{n(p-1)}(R)$ is $G$-invariant.

We now combine this result with $(*)$. Take any $y \in L_{p} \backslash G$. Then (as $L_{p}$ and $G$ are restricted),

$$
J:=\sum_{j \geq 0}(\operatorname{ad} y)^{j}\left(R^{\prime} \otimes F x_{1}^{p-1} \cdots x_{n}^{p-1}\right)=\sum_{j=0}^{p-1}(\operatorname{ad} y)^{j}\left(R^{\prime} \otimes F x_{1}^{p-1} \cdots x_{n}^{p-1}\right) .
$$

From this one proves by $(*)$ that $J \subset Q_{n(p-1)-(p-1)} . J$ is invariant under $y$ by construction and by induction it is seen that $J$ is $G$-invariant. This implies $(n-1)(p-1) \leq 0$ and therefore $n=1$.

Theorem 7. Let $L$ be an indecomposable Lie algebra having a faithful irreducible representation $\rho: L \rightarrow g l(U)$ of dimension $<p^{2}$. Then $S(L)$ is one of the following:

(1) $S(L)=C(L) \oplus S_{2}, S_{2}$ simple.

(2) $S(L)=S_{1}, C(L) \subset S_{1}^{(1)}, S_{1}^{(1)}=S_{1}, S_{1} / C(L)$ simple.

(3) $S(L)=S_{1} \oplus\left(S^{\prime} \otimes A(1 ; 1)\right), S_{1}$ abelian, $S^{\prime}$ is semisimple and has a faithful irreducible representation of dimension $<p$.

(4) $S(L)=\operatorname{rad}(L)$.

Proof. Put $S(L)=S_{1} \oplus \cdots \oplus S_{r}$. Let $U_{0}$ be an irreducible $S(L)$-submodule of $U$. 
(a) Consider first the case that $\operatorname{dim} \widetilde{U_{0}(S(L))} \geq p$. (I.3) proves that $U_{0}$ is a faithful $S(L)$-module. (I.1) shows that we have for any irreducible $S_{i}$-module $V_{i}$ of $U_{0}$

$$
U_{0} \cong \bigotimes V_{i}
$$

Since $U_{0}$ is faithful, all $V_{i}$ are faithful $S_{i}$-modules. If $r=1$ we are done by (II.5). So assume $r>1$. If, for some $j>1, \operatorname{dim} V_{j}<p$, then (II.1, Corollary) shows $L=S_{j}$ and $C(L)=0$. In this case $S_{j}$ is a minimal ideal of $L$, proving that $S_{j}$ is simple. This is case (1).

If $\operatorname{dim} V_{j} \geq p \forall j \geq 2$ then $r=2$ and $\operatorname{dim} V_{1}<p$. (II.1, Corollary) proves $\operatorname{dim} S_{1} \leq 1$. Then $S_{1} \subset C(S(L))$ and $S_{1}$ acts as scalars on the irreducible $S(L)$-module $U_{0}$. Hence $\widetilde{V_{1}\left(S_{1}\right)} \supset U_{0}$ and $\operatorname{dim} \widetilde{V_{1}\left(S_{1}\right)} \geq p$. According to (I.4) this implies $S_{1}=C(L)$. (II.5) shows that $S_{2}$ is simple.

(b) Consider the case that $\operatorname{dim} \overparen{U_{0}(S(L))}<p$. Let $V_{i}$ be irreducible $S_{i}$ -

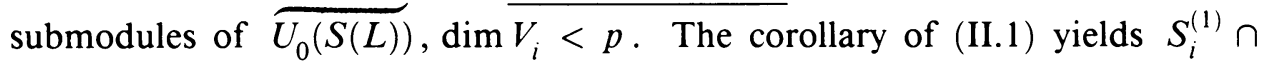
$C(L)=0$, proving (by definition of $S(L)$ ) that $S_{1}=\operatorname{rad}(L)$. Then $S_{1}$ is solvable, and hence $\operatorname{dim} V_{1}$ is a $p$-power. We conclude $\operatorname{dim} V_{1}=1$ and $S_{1}^{(1)}$ annihilates $V_{1}$. (I.4) yields $S_{1}^{(1)}=0$.

If, for some $j>1, S_{j}=L$ then $S_{j}$ is simple. This is case (1). We may therefore assume that $S_{j} \neq L$ for all $j>1$. According to Lemma 5 there are simple algebras $S_{j}^{\prime}$ such that $S_{j}=S_{j}^{\prime} \otimes A(1 ; \mathbf{1}) . V_{j}$ is a faithful irreducible $S_{j}^{\prime}$-module. Put $S^{\prime}=\sum S_{j}^{\prime}$.

While cases (1)-(3) of Theorem 7 give considerable information about $S(L)$ and by this describes $L$ in terms of smaller pieces, case (4) allows further reduction.

Theorem 8. Let $L$ be an indecomposable Lie algebra having a faithful irreducible representation $\rho: L \rightarrow g l(U)$ of dimension $<p^{2}$. Assume that $S(L)=\operatorname{rad}(L)$. Then either $\operatorname{dim} L=1$ or $L$ has an abelian ideal $I \not \subset C(L)$ or $L$ is the six-dimensional split extension of $s l(2)$ by the three-dimensional Heisenberg algebra.

Proof. (a) Assume that $C(L)$ is the only abelian ideal of $L$. If $S(L)=C(L)$ then by definition $L / C(L)$ is a semisimple algebra having no minimal nonabelian ideal, i.e. $L / C(L)=(0)$. We may therefore assume that $\operatorname{rad}(L) \neq$ $C(L)$. Since $C(L)$ is assumed to be the only abelian ideal we have $C(L) \neq 0$. In this situation we can apply the methods developed in [13] as follows.

(b) Let $I$ be the ideal of $L$ maximal with the property $I^{(1)} \subset C(L)$ and take $U_{0}$ to be an irreducible $I$-submodule. Since $I$ is nonabelian, we have $\operatorname{dim} U_{0} \geq p$ and hence $U_{0}$ is faithful (I.3). This implies $\operatorname{dim} C(I)=1$ and hence $C(I)=C(L)$. Consider the bilinear mapping

$$
\alpha: I \times I \rightarrow C(L) \cong F
$$


given by $\alpha(x, y)=[x, y]$. Put $J:=\operatorname{rad}(\alpha)=\{x \in I \mid \alpha([x, I])=0\}$. Since by definition of $\alpha J \subset C(I)=C(L), \alpha$ is nondegenerate on $I / C(L) \times I / C(L)$. Note that $[x, y]$ acts nilpotently on $U_{0}$ if $\alpha([x, y])=0$ and invertibly otherwise. General representation theory of nilpotent algebras now proves

$$
\operatorname{dim} U_{0} \geq p^{(1 / 2) \operatorname{dim} I / C(L)},
$$

hence $\operatorname{dim} I / C(L) \leq 2$. As $I$ is not abelian, we obtain $\operatorname{dim} I=3$ and $I$ is a Heisenberg algebra.

(c) Put $K:=\{x \in L \mid[x, I]=0\} . K$ is an ideal of $L$. Let $V$ be an irreducible $(K+I)$-submodule of $U$. As any $I$-submodule has dimension $\geq p$, $V$ is a faithful irreducible $(K+I)$-module (I.3). According to (I.1) $K$ has a faithful irreducible module of dimension $<p$. Then (II.1, Corollary) implies $\operatorname{dim} K=1$. Therefore $(I+K)^{(1)} \subset I^{(1)} \subset C(L)$. The maximality of $I$ yields $K \subset I$ and hence $K \subset C(I)=C(L)$.

(d) Let $M$ be any ideal of $L$ such that $[M, I] \subset C(L) . \alpha$ can be extended to

$$
\alpha^{\prime}: M \times I \rightarrow C(L) \cong F, \quad \alpha^{\prime}(x, y)=[x, y] .
$$

Since $\alpha$ is nondegenerate on $I / C(L) \times I / C(L), M$ decomposes

$$
M=K+I, \quad[K, I]=0 .
$$

According to (c) $K=C(L)$, and hence $M=I$.

(e) Let $R$ be an ideal of $L$ such that $R^{(1)} \subset I$. Consider the representation

$$
\mu: L \rightarrow g l(I / C(L)) \text {. }
$$

Since $I / C(L)$ is two-dimensional and $L$ has no abelian ideals except $C(L)$, $I / C(L)$ is an irreducible $L$-module. The property $R^{(1)} \subset \operatorname{ker}(\mu)$ and (I.4) ensure the existence of $E \in R^{*}$ such that

$$
\mu(x)-E(x) \text { id is nilpotent for all } x \in R .
$$

Then $(\operatorname{ad} x)^{p}(y)-E(x)^{p} y \in C(L) \forall x \in R, y \in I$ and

$$
0=(\operatorname{ad} x)^{p}\left(\left[y_{1}, y_{2}\right]\right)=2 E(x)^{p}\left[y_{1}, y_{2}\right] \quad \forall x \in R, y_{1}, y_{2} \in I .
$$

Since $I^{(1)} \neq 0$, this yields $E(x)=0 \forall x \in R . R$ acts nilpotently on $I / C(L)$, proving $R \subset \operatorname{ker}(\mu)$. Then $[R, I] \subset C(L)$. From (d) we obtain $R \subset I$. As a result, $L / I$ acts faithfully on the two-dimensional irreducible module $I / C(L)$ and is semisimple. This is possible only if $L / I \cong \operatorname{sl}(2)$. Considering eigenvalues of some semisimple element in $\operatorname{sl}(2)$, we conclude that the extension splits.

The representation theory of this six-dimensional split extension algebra is completely developed in [13].

Theorem 9. Let $L$ be an indecomposable Lie algebra having a faithful irreducible representation $\rho: L \rightarrow g l(U)$ of dimension $<p^{2}$. Put

$$
K:=\{x \in L \mid[x, S(L)]=0\} .
$$

Then $K=C(L)$ or $K=\operatorname{rad}(L)$ is abelian. 
Proof. Assume that $C(L) \varsubsetneqq K$. Put as above $S(L)=S_{1} \oplus \cdots \oplus S_{r}$. Since $S_{i}$ is (for $i \geq 2$ ) a minimal nonabelian ideal of $L$ and $K$ centralizes $S_{i}$, we have $K \cap S_{i}=(0) \quad(i=2, \ldots, r)$. By construction of the socle then $K / C(L)$ intersects $S_{1} / C(L)$ nontrivially. Hence $I:=K \cap S_{1}$ is a noncentral abelian ideal of $L$. Let $U_{0}$ be an irreducible $I$-submodule. As $I \not \subset C(L),(I .4(4))$ proves that $\operatorname{dim} \widetilde{U_{0}(I)}<p . \widetilde{U_{0}(I)}$ is a $K$-module because $[I, K] \subset\left[S_{1}, K\right]=0$. $K^{(1)} \cap S_{1}$ is contained in $C(K)$ and therefore acts nilpotently on any irreducible $K$-submodule of $\widetilde{U_{0}(I)}$. Then $K^{(1)} \cap S_{1}$ vanishes according to (I.4). As a result, $K^{(1)} \cap S(L)=0$ and therefore $K$ is abelian. This in turn implies by definition that $S_{1}=\operatorname{rad}(L)$ and $I=K$. Then $\widetilde{U_{0}(I)}$ is a $\operatorname{rad}(L)$-module of dimension $<p$, proving that $\operatorname{rad}(L)$ is abelian and $K=\operatorname{rad}(L)$.

This theorem shows that $K=C(S(L))$ is contained in $S(L)$ and $\dot{L} / K$ may be considered a subalgebra of $\operatorname{Der}(S(L))$. Therefore $L$ is described in terms of its socle only.

Combining the aforementioned results we obtain some structural insight for the general case.

Theorem 10. Let $L$ be a Lie algebra having a faithful irreducible representation of dimension $<p^{2}$. Then $L=\bigoplus_{i=1}^{s} L_{i}$ where for $s>1, L_{2}, \ldots, L_{s-1}$ are simple and $L_{s}$ is simple or $L_{s}=C(L)$. They all have a faithful irreducible representation of dimension $<p . L_{1}$ is indecomposable having a faithful irreducible representation of dimension $<p^{2}$.

Any CSA $H$ of $L$ decomposes $H=\bigoplus\left(H \cap L_{i}\right)$, and $H \cap L_{i}$ is a CSA of $L_{i}$. Any ideal $J$ of $L$ decomposes $J=\bigoplus\left(J \cap L_{i}\right)$.

Proof. Decompose $L=\bigoplus_{i=1}^{s} L_{i}$ into indecomposable ideals. According to (I.1) all summands, except possibly one, have faithful irreducible representations of dimension $<p$. In particular, all derivations of these are inner. This observation immediately yields that $H$ and $J$ are decomposable as wanted and that $H \cap L_{i}$ is a CSA of $L_{i}$.

\section{APPLICATIONS TO CLASSIFICATION THEORY}

In [6] of Block-Wilson, in which they classified the restricted simple Lie algebras, the problem which we have discussed in this note occurs in the following manner. Starting with a restricted simple Lie algebra $L$, they consider twosections $A=\sum L_{i \alpha+j \beta}$ where $\alpha, \beta$ are $G F(p)$-independent roots with respect to some torus. These algebras are restricted, every torus has rank at most two and every CSA acts triangulably on $A$. They choose some particular torus ("optimal") $T$ of $A$ and some maximal subalgebra $A_{0}$ ("distinguished") which contains the CSA $C_{A}(T)$. By means of an irreducible $A_{0}$-submodule of $A / A_{0}$ one defines a standard filtration and considers the associated graded algebra $G$. $G$ inherits the following properties from $A$ : There is some restricted subal- 
gebra $G_{0}$ which contains a CSA $C_{G_{0}}(T)$ of $G . T$ then is a maximal torus of $G_{0}$ of dimension 2 and $C_{G_{0}}(T)$ acts triangulably on $G$. In addition, $G_{0}$ acts faithfully and irreducibly on $G_{-1}$. In Chapter 7 of [6] Block-Wilson deal with the case that $G_{0}$ has toral rank 1 with respect to $T$ (or equivalently, with respect to the CSA $\left.C_{G_{0}}(T)\right)$ and $\operatorname{dim} G_{-1} \leq 7 p<p^{2}-3 p$.

Similarly, the above-mentioned problem occurs in some forthcoming paper of Benkart-Osborn [2]. They start with a CSA $H$ of a simple (nonrestricted) Lie algebra $L$ and consider a two-section $K=\sum L_{i \alpha+j \beta}$ where $\alpha, \beta$ are roots with respect to $H$ which are $G F(p)$-independent but $F$-dependent. They, too, consider a graded algebra $G$, obtained in the same way as Block-Wilson, and obtain in [2] Lemma $3.3(\mathrm{iv}(\mathrm{b}))$ as a major case

$$
\begin{aligned}
& G_{0} \text { acts faithfully on } G_{-1} ; \operatorname{dim} G_{-1}=p^{2}-1, \\
& G_{0} \text { is semisimple. }
\end{aligned}
$$

Our present results yield with only little extra work that $G_{0}$ is indecomposable and $S\left(G_{0}\right)$ is simple, thereby reducing this case to a very specific situation:

Remark. Assume that $L$ is semisimple and suppose that $\rho: L \rightarrow g l(U)$ is a faithful irreducible representation of dimension $p^{2}-1$. Let $H$ be a CSA of $L$ such that $L=\sum_{i \in F} L_{i \alpha}(H)$ is the root space decomposition with respect to $H$. Assume that $\rho\left(H^{(1)}\right)$ consists of nilpotent transformations. Then

(1) $L$ is indecomposable.

(2) $S(L)$ is simple.

(3) $L \subset \operatorname{Der}(S(L))$.

Proof. (a) Since $\rho$ is faithful and $H^{(1)}$ acts by nilpotent transformations on $U, H^{(1)}$ acts nilpotently on $L$. Then $\alpha(h)=0 \quad \forall h \in H^{(1)}$. Therefore $\alpha$ is linear. Put $H_{0}=\operatorname{ker}(\alpha)$. As $H^{(1)} \subset H_{0}, H_{0}$ is an ideal of $H$ of codimension 1.

Let $I, J$ be ideals of $L$ and suppose that $I \cap H \not \subset H_{0}$. Then $\left[L_{i \alpha}, I \cap H\right]=$ $L_{i \alpha} \cap I \quad \forall i \neq 0$, proving

$$
J \subset H+I \cap J .
$$

(b) Suppose $L=\bigoplus_{i=1}^{s} L_{i}$. According to (II.10) $H \cap L_{i}$ is a CSA of $L_{i}$. If $L_{i} \cap H \subset H_{0}$, then $L_{i} \cap H$ acts nilpotently on $L_{i} . L_{i}$ is therefore nilpotent, contradicting $\operatorname{rad}(L)=0$. We conclude $L_{1} \cap H \not \subset H_{0}$. According to the above remark $L_{i} \subset H \forall i>1$, and $L_{i} \subset \operatorname{rad}(L)=0$. Therefore $L$ is indecomposable.

(c) Let $U_{0}$ be an irreducible $S(L)$-module. Since $\operatorname{dim} U$ is not divisible by $p, U$ is not induced by $\overparen{U_{0}(S(L))}$. Therefore $\operatorname{dim} \overparen{U_{0}(S(L))} \geq p$. In combination with $\operatorname{rad}(L)=0$ the proof of (II.7) shows that $S(L)=S_{2}$ is simple. Theorem 9 implies (3).

To deal with the former application we will consider the following situation.

Let $H$ be a CSA of $L$ and $L=\sum L_{\alpha}(H)$ be the root space decomposition of $L$ with respect to $H . L$ is said to have toral rank one with respect to $H$ 
if the roots span a one-dimensional $G F(p)$-vector space. Note that in this case all roots are contained in $G F(p) \alpha$ for any nonzero root $\alpha$. This in turn means that an element $h \in H$ acts nilpotently on $L$ if $\alpha(h)=0$ for some nonzero root $\alpha$.

Assumption B. Suppose that $H$ is a CSA of $L$ such that $L$ has toral rank one with respect to $H$. Assume that $L$ has a faithful irreducible representation $\rho: L \rightarrow g l(U)$ of dimension $<p^{2}$. In addition, $\rho\left(H^{(1)}\right)$ is supposed to consist of nilpotent transformations.

Let $H$ be a CSA of $L$ such that $L$ has toral rank 1 with respect to $H$. Then Theorems (II.7) and (II.10) reduce to a much simpler structure. We refer to [14] for the following.

Theorem 1. Let $L$ be a Lie algebra of toral rank 1 with respect to some CSA $H$. Assume that $H^{(1)}$ acts nilpotently on $L$.

Let $J$ be a nonsolvable ideal of $L$. Then

(1) $L=J+H . J \cap H$ is a CSA of $J . J$ has toral rank 1 with respect to $J \cap H$.

(2) Any ideal $I$ of $L$ can be written as $I=I \cap J+I \cap H$.

Corollary. Let $L$ be a Lie algebra satisfying (B). Then $L=L_{1} \oplus L_{2}$ where $L_{1}$ is indecomposable and $L_{2} \subset C(L)$.

One of the following is true:

(1) $L / C(L)$ is simple or $\operatorname{dim} L=1$.

(2) $L$ is the six-dimensional split extension of $s l(2)$ by a Heisenberg algebra.

(3) $L$ has an abelian ideal $I \not \subset C(L)$.

Proof. According to (II.10) $L=\bigoplus_{i=1}^{s} L_{i}$ decomposes, and if $s>1, L_{s}$ is not solvable. Put in Theorem (III.1) $J:=L_{s}$ and $I:=\bigoplus_{i=1}^{s-1} L_{i}$. Then $I \subset H$ (as $I \cap J=0$ ). Therefore $I^{(1)}$ is an ideal acting nilpotently on $U$. Hence $I^{(1)}=0$, whence $[I, L] \subset I^{(1)}+[I, J]=0$ and $I \subset C(L)$. This proves the first assertion.

If $\operatorname{rad}(L)=C(L)$, then $L / C(L)$ is simple (III.1). Therefore we may assume that $\operatorname{rad}(L) \neq C(L)$ and $C(L)$ is the only abelian ideal of $L$. Note that in this case $C(L) \subset \operatorname{rad}(L)^{(1)} \subset L_{1}$ and therefore $L$ is indecomposable. Since $\operatorname{rad}(L)$ is a direct summand in $S(L)$ and is not abelian, only case (4) in (II.7) can occur. Then (II.8) gives the result.

In order to determine the full structure of $L$ we first deal with the case that $L$ has a noncentral abelian ideal. Let $L_{p}$ denote the $p$-envelope of $\rho(L)$ in $g l(U)$.

Proposition 2. Assume that $L$ satisfies (B) and that $L$ has an abelian ideal $I \not \subset C(L)$. Then there is a restricted homomorphism $\varphi: L_{p} \rightarrow W(1 ; \mathbf{1})$, such that $\varphi\left(L_{p}\right)$ is an essential subalgebra of $W(1 ; \mathbf{1})$ and $J:=\operatorname{ker}(\varphi)$ is abelian. Put $G:=\varphi^{-1}\left(W(1 ; \mathbf{1})_{(0)}\right)$. Then $[G, J]$ acts nilpotently on $U$. 
Proof. We use the notation of (I.2) and (I.4). Since $I \not \subset C(L)$ we have $L_{p} \neq$ $G\left(I, U_{0}\right)=: G$ and hence

$$
U \cong U\left(L_{p}, S\right) \otimes_{U(G, S \mid G)} \widetilde{U_{0}(I)} .
$$

As a result, $\operatorname{dim} L_{p} / G=1$. Let $J$ denote the maximal ideal of $L_{p}$ in $G$. (I.6) yields the existence of a restricted homomorphism

$$
\varphi: L_{p} \rightarrow W(1 ; \mathbf{1})
$$

such that $\varphi^{-1}\left(W(1 ; \mathbf{1})_{(0)}\right)=G\left(I, U_{0}\right)$. By definition $[G, I]$ acts nilpotently on $U$. We next want to prove that $J$ is abelian.

If $J$ is not solvable, then neither is $J^{(1)} \subset L$. According to (III.1) this implies $L=H+J^{(1)}$. Consider the root space decomposition with respect to $H$. Since $L$ and $J^{(1)}$ have toral rank 1 with respect to $H$ and $H \cap J^{(1)}$, respectively, there is an $h \in H \cap J^{(1)}$ and a root $\alpha$, such that $\alpha(h) \neq 0$. Hence

$$
\sum_{i \neq 0} I_{i \alpha} \subset[I, J] \subset[I, G] \subset \operatorname{ker}(E) .
$$

Since $H^{(1)}$ acts nilpotently on $U$ this yields

$$
[I, L] \subset[I, H]+\left[I, J^{(1)}\right] \subset \sum_{i \neq 0} I_{i \alpha}+H^{(1)} \cap I+\left[I, J^{(1)}\right] \subset \operatorname{ker}(E),
$$

hence $L \subset G$ and $L_{p} \subset G$, a contradiction. Therefore $J$ is solvable. $U_{0}(I)$ is a $J$-module of dimension $<p$. As every irreducible $J$-module has dimension a $p$-power, there is a one-dimensional $J$-submodule. This is annihilated by $J^{(1)}$. (I.4) yields $J^{(1)}=0$. Choose some one-dimension $J$-submodule $V_{0}$ of $U$. As above $G\left(J, V_{0}\right) \neq L_{p}$ and $\operatorname{dim} L_{p} / G\left(J, V_{0}\right)=1$. On the other hand, we have $I \subset J$ and by definition $G\left(I, U_{0}\right) \supset G\left(J, V_{0}\right)$. This proves equality. By definition, $\left[G\left(J, V_{0}\right), J\right]$ acts nilpotently on $U$.

We apply this result and use in the next theorem the same notation.

Theorem 3. Assume that $L$ satisfies (B) and that $L$ has an abelian ideal I $\not \subset$ $C(L)$. Then $L$ is one of the following:

(1) $L=F h \oplus \bigoplus_{i \in S \subset G F(p)} F y_{i},\left[h, y_{i}\right]=i y_{i},\left[y_{i}, y_{j}\right]=0, \rho\left(y_{i}\right)$ is invertible for all $i, S \backslash\{0\} \neq \varnothing$.

(2) $L=F x \oplus \bigoplus_{i=0}^{k} F y_{i},\left[x, y_{i}\right]=y_{i-1},\left[y_{i}, y_{j}\right]=0,1 \leq k \leq p-1$, $F y_{0}=C(L) \neq(0)$.

(3) $L=F h \oplus F x \oplus \bigoplus_{i=0}^{k} F y_{i},\left[h, y_{i}\right]=i y_{i},[h, x]=-x,\left[x, y_{i}\right]=y_{i-1}$, $\left[y_{i}, y_{j}\right]=0,1 \leq k \leq p-1 . \rho\left(y_{i}\right)$ is nilpotent for $1 \leq i \leq k$.

$(4) L$ is the split extension of $W(1 ; \mathbf{1})$ by the restricted $W(1 ; \mathbf{1})$-module $A(1 ; \mathbf{1})$.

(5) $L$ is the split extension of $s l(2)$ (considered as an essential subalgebra of $W(1 ; \mathbf{1}))$ by $A(1 ; \mathbf{1})$.

Proof. We prove first that (1)-(5) of the theorem are true for $L_{p}$ instead of $L$ and finally will show that the same results are true for $L$. 
(1) Assume first that $L_{p} / J$ is one-dimensional and not [p]-nilpotent. Then there exists a toral element $h \in L_{p} \backslash J$ (which then acts semisimple on $J$ ). Decompose $J=\sum J_{i}$ into root spaces with respect to ad $\left.h\right|_{J}$. Since this is a semisimple endomorphism, $J_{i}$ is in fact the eigenspace. Since $J$ is abelian, $J$ admits an eigenvalue function $E \in J^{*}$ and $J_{i} \cap \operatorname{ker}(E)$ is an ideal of $L_{p}$. This acts nilpotently on $U$, and therefore is zero. As a result, $\operatorname{dim} J_{i} \leq 1$. This is case (1).

(2) If the essential subalgebra $\varphi\left(L_{p}\right)$ is not of the above type we have

$$
\varphi\left(L_{p}\right)=F \bar{x} \oplus \varphi(G), \quad \bar{x}^{[p]}=0 .
$$

If moreover $\varphi(G) \neq 0$, we even may choose elements $h \in G$ and $x \in L_{p} \backslash G$ such that $h^{[p]}=h, \bar{x}^{[p]}=0$ and $[h, x]=-x$ (observe that $\operatorname{ad} h$ is a semisimple endomorphism).

Consider $J^{\prime}:=\left\{y \in J \mid(\operatorname{ad} x)^{j}(y) \in \operatorname{ker}(E) \forall j>0\right\}$. Since $[G, J] \subset \operatorname{ker}(E)$ and $L_{p}=F x+G, J^{\prime}$ is an ideal of $L_{p}$ satisfying $\left[L_{p}, J^{\prime}\right] \subset \operatorname{ker}(E)$. As a result, $J^{\prime}=C\left(L_{p}\right)$.

(3) Consider the nilpotent subalgebra $K:=J+F x$ of $L_{p}$. Let $V$ be an irreducible $K$-submodule of $U$ and $R$ the kernel of this representation. Note that $R \cap J \subset J^{\prime}$. According to (2) we obtain

$$
R \cap J \subset C\left(L_{p}\right) \cap \operatorname{ker}(E)=0 .
$$

The assumption $R \neq 0$ would therefore imply $K=R+J$ and hence $K^{(1)} \subset$ $R^{(1)}+R \cap J+J^{(1)} \subset R$. But then $[x, J] \subset \operatorname{ker}(E)$ which leads to the contradiction $x \in G$. The $K$-module $V$ is therefore faithful.

Decompose $J=\bigoplus J_{i}$ into indecomposable $F x$-modules. As $J$ is abelian, every $J_{i}$ is an ideal of $K$ and as such it intersects $C(K)$ nontrivially. Since $V$ is faithful irreducible, we have $\operatorname{dim} C(K) \leq 1$. Then $J$ itself is indecomposable. The Jordan normal form of ad $x \mid J$ provides a basis $\left(y_{0}, \ldots, y_{k}\right)$ for $J$ with $\left[x, y_{i}\right]=y_{i-1}$. Note that according to (2) $F y_{0}=C\left(L_{p}\right) \neq 0$. Since $\bar{x}^{[p]}=0$ we also have $(\operatorname{ad} x)^{p} \mid J=0$, proving $k \leq p-1$. The observation that $[x, J] \not \subset \operatorname{ker}(E)$ yields $k \geq 1$.

(4) If $\varphi\left(L_{p}\right)=F \bar{x}$ the above shows that we are in case (2). If $\varphi\left(L_{p}\right)=F \bar{x} \oplus$ $F \bar{h}$ we have in addition (see (2)) $[h, x]=-x$. Then any $y_{i}$ is an eigenvector with respect to ad $h$. As $\left[h, y_{0}\right] \in[h, C(L)]=0$ this yields $\left[h, y_{i}\right]=i y_{i}$. We also see that for $i \neq 0 \quad y_{i} \in[G, J] \subset \operatorname{ker}(E)$. This settles the case $\operatorname{dim} L_{p} / J \leq$ 2 .

(5) We end up with: $\varphi\left(L_{p}\right)$ is isomorphic to $s l(2)$ or $W(1 ; \mathbf{1})$. Applying the results of (3) we obtain that $C\left(L_{p}\right) \neq 0 . J / C\left(L_{p}\right)$ is a restricted $\varphi\left(L_{p}\right)$-module of dimension $k<p$. Computing traces one gets

$$
0=\operatorname{trace}(\operatorname{ad} h \mid J)=\sum_{i=1}^{k} i=\frac{k(k+1)}{2},
$$


i.e. $k=p-1$. A direct computation shows that there is a realization $J \cong$ $A(1 ; \mathbf{1})$ and $L_{p} / J \cong W(1 ; \mathbf{1})$ or $L_{p} / J \cong F \partial \oplus F x \partial \oplus F x^{2} \partial$.

(6) It remains to prove that the extension

$$
0 \rightarrow J \rightarrow L_{p} \rightarrow L_{p} / J \rightarrow 0
$$

splits.

Consider first the case that $L_{p} / J \cong \operatorname{sl}(2)$. Since ad $h$ is semisimple, there is an eigenvector $y \in L_{p} \backslash J$ such that $u=[x, y]-h \in J,[h, y]=y$. Therefore $[h, u]=0$ proving that $u \in C\left(L_{p}\right)$. Put $h^{\prime}:=h+\frac{1}{2} u . F x+F h^{\prime}+F y$ is isomorphic to $s l(2)$. The extension splits.

Next consider $L_{p} / J \cong W(1 ; \mathbf{1})$. Using the former result we put $e_{-1}:=x$, $e_{0}:=h, e_{1}:=y$. There are elements $e_{2}, \ldots, e_{p-2} \in L_{p} \backslash J$ with the properties $\left[e_{-1}, e_{i}\right]=(i+1) e_{i-1},\left[e_{0}, e_{i}\right]=i e_{i}$. One proves inductively $\left[e_{i}, e_{j}\right]=(j-$ i) $e_{i+j}$ (for $\left.1 \leq i, j, i+j \leq p-2\right)$ and $=0$ if $i+j>p-2$. The extension splits.

(7) We now derive the structure of $L$. Note that $L_{p}^{(1)} \subset L$. In cases (4) and (5) this shows $L=L_{p}$. Assume in the other cases $L \neq L_{p}$. Case 1. Since $I \not \subset C(L), L$ is not abelian. Then there exist $\alpha, \beta \in F, \alpha \neq 0$, such that $h^{\prime}:=\alpha h+\beta y_{0} \in L$. Then $L=F h^{\prime}+\sum F y_{i}$ having the same structure. Case 2 . There exist $\alpha, \beta \in F$ such that $x^{\prime}:=\alpha x+\beta y_{k} \in L$. Then $L=F x^{\prime} \oplus \bigoplus_{i=0}^{k} F y_{i}$ having the same structure. Case 3. $L=L_{p}^{(1)}=F x \oplus \bigoplus_{i=0}^{k} F y_{i}$. Then $L$ acts nilpotently on $x$, but $L_{p}$ does not, a contradiction.

For all algebras occurring in Theorem $3 A(1 ; 1)$ is a faithful irreducible module, as follows:

Case 1. $\rho(h)=(1+x) \partial, \rho\left(y_{i}\right)\left(x^{j}\right)=(1+x)^{i} x^{j}$.

Case $2-5$. $L$ may be considered as a subalgebra of the semidirect sum $W(1 ; 1)$ $+A(1 ; \mathbf{1})$, and then containing $\partial \in W(1 ; \mathbf{1}) \cap L . A(1 ; \mathbf{1})$ is an irreducible $L$ module by restricting the representation

$$
\begin{aligned}
& \rho: W(1 ; \mathbf{1})+A(1 ; \mathbf{1}) \rightarrow g l(A(1 ; \mathbf{1})), \\
& \rho\left(x^{i} \partial+x^{j}\right)\left(x^{k}\right):=x^{i} \partial\left(x^{k}\right)+x^{j+k}
\end{aligned}
$$

to $L$.

Theorem 4. Assume that $L$ satisfies (B) and that $S(L) / C(L)$ is simple. Then $L / C(L)$ can be embedded into $\operatorname{Der}(S(L) / C(L))$. The following cases can occur:

(1) $L=\operatorname{sl}(2) \oplus C(L)$,

(2) $L=W(1 ; \mathbf{1}) \oplus C(L)$,

(3) $W(1 ; 2) \oplus C(L) \subset L \subset \operatorname{Der}(W(1 ; 2) \oplus C(L))$,

(4) $S(L) / C(L) \in\left\{H(2 ; \mathbf{1} ; \phi)^{(2)}, H(2 ;(2,1) ; \phi)^{(2)}\right\}$.

Proof. Put $I:=\{x \in L \mid[x, S(L)] \subset C(L)\}$. Since $S(L)$ is not solvable, (III.1) yields that $I=I \cap S(L)+I \cap H$. The simplicity of $S(L) / C(L)$ shows $I \subset \operatorname{rad}(L)$. By definition of $S(L)$ we have $\operatorname{rad}(L)=C(L)$. Then the adrepresentation defines an embedding $L / C(L) \rightarrow \operatorname{Der}(S(L) / C(L))$. 
From [16] we conclude that $S(L) / C(L)$ is one of the following simple algebras:

$$
s l(2), W(1 ; \mathbf{1}), W(1 ; \mathbf{n})(n \geq 2), H(2 ; \mathbf{n} ; \phi)^{(2)} .
$$

(1) $S(L) / C(L) \cong s l(2)$ : Then $L / C(L) \cong s l(2)$ and $L=s l(2) \oplus C(L)$.

(2) $S(L) / C(L) \cong W(1 ; \mathbf{1})$ : Then $L / C(L) \cong \operatorname{Der}(W(1 ; \mathbf{1})) \cong W(1 ; \mathbf{1})$.

It is proved in [13] that the faithful irreducible modules of the nonsplit central extension of $W(1 ; \mathbf{1})$ have dimension $\geq p^{p-1 / 2}$ which is at least $p^{2}$ (as $\left.p>3\right)$. Therefore the extension splits.

(3) $S(L) / C(L) \cong W(1 ; \mathbf{n})(n \geq 2)$. [8] shows that $n=2$ and $S(L)=$ $S_{1} \oplus C(L)$ splits. Consider $S(L) \subset L \subset g l(U)$ and let $S(L)_{p}, L_{p}$ denote the $p$-envelopes in $g l(U)$. Note that since $S_{1}$ is not restrictable $S(L)_{p}=$ $\left(S_{1}\right)_{p} \oplus C(L)=\operatorname{Der}(W(1 ; 2)) \oplus C(L)$. Then $L_{p}=\operatorname{Der}(W(1 ; 2)) \oplus I$ where $I:=\left\{x \in L_{p} \mid\left[x, S_{1}\right]=0\right\} . I^{(1)} \subset L$ centralizes $S_{1}$ and as above we conclude that $I^{(1)} \subset C(L)$ and hence $I$ is nilpotent. Application of (II.10) to $L_{p}$ yields $I=C\left(L_{p}\right)$.

(4) $S(L) / C(L)=H(2 ; \mathbf{n} ; \phi)^{(2)}$ : A simple dimension argument yields

$$
\operatorname{dim} S(L) \leq \operatorname{dim} g l(U) \leq\left(p^{2}-1\right)^{2} \leq p^{4}-p .
$$

Therefore $\mathbf{n}=(1,1)$ or $\mathbf{n}=(2,1)$.

It seems reasonable that also in (4) the central extension splits and $L \rightarrow$ $\operatorname{Der}\left(H(2 ; 1 ; \phi)^{(2)}\right) \oplus C(L)$ is true. A proof of that, however, seems to involve an awful mass of computations and yields only comparatively little additional information.

In cases (1)-(3) there exist faithful irreducible modules $M$ of dimension 2, $M \cong W(1 ; \mathbf{1})$ of dimension $p, M \cong A(1 ; 2) / F$ of dimension $p^{2}-1$, respectively. Here we consider $C(L)$ acting as $F$ id on these modules if $C(L) \neq 0$.

\section{REFERENCES}

1. G. Benkart and J. M. Osborn, Toral rank one Lie algebras, J. Algebra 115 (1988), 238-250.

2. __ Simple Lie algebras of characteristic $p$ with dependent roots, preprint.

3. R. E. Block, Trace forms on Lie algebras, Canad. J. Math. 14 (1962), 553-564.

4. R. E. Block and H. Zassenhaus, The Lie algebras with a non-degenerate trace form, Illinois J. Math. 8 (1964), 543-549.

5. R. E. Block, Differentiably simple algebras, Bull. Amer. Math. Soc. 74 (1968), 1086-1090.

6. R. E. Block and R. L. Wilson, Classification of the restricted simple Lie algebras, J. Algebra 114 (1988), 115-259.

7. J. Dixmier, Algèbre enveloppantes, Gauthier-Villars, 1974.

8. A. S. Dzhumadil'daev, Central extensions of the Zassenhaus algebra and their irreducible representations, Math. USSR Sb. 54 (1986), 457-474.

9. R. Farnsteiner and H. Strade, On the derived length of solvable Lie algebras, Math. Ann. 282 (1988), 503-511.

10. J. B. Jacobs, On classifying simple Lie algebras of prime characteristic by nilpotent elements, J. Algebra 19 (1971), 31-50. 
11. A. I. Kostrikin, Theorem on semisimple Lie-p-algebras, Mat. Zametki 2 (1967), 465-474.

12. H. Strade, Lie algebra representations of dimension $p-1$, Proc. Amer. Math. Soc. 41 (1973), 419-424.

13. $\_$, Zur Darstellungstheorie von Lie-Algebren, Abh. Math. Sem. Univ. Hamburg 52 (1982), 66-82.

14. __ Cyclically graded Lie algebras (in preparation).

15. H. Strade and R. Farnsteiner, Modular Lie algebras and their representations, Textbooks and Monographs, vol. 116, Dekker, 1988.

16. R. L. Wilson, Simple Lie algebras of toral rank one, Trans. Amer. Math. Soc. 236 (1978), 287-295.

Department of Mathematics, University of Wisconsin, Madison, Wisconsin 53705

Department of Mathematics, University of Hamburg, Bundesstrasse 55, 2 Hamburg 13, Federal Republic of Germany (Current address) 